\title{
Expectativas del paciente ante la artroplastia total de rodilla.
}

\author{
DOI: http://dx.doi.org/10.37315/SOTOCAV2020281551 \\ FERRANDO-MESEGUER EJ, MÍNGUEZ-REY MF, MIFSUT-MIEDES D, SILVESTRE-MUÑOZ A, GOMAR-SANCHO F. \\ SERVICIO DE CIRUGÍA ORTOPÉDICA Y TRAUMATOLOGÍA, HOSPITAL CLÍNICO-MALVARROSA DE VALENCIA.
}

UNIVERSITAT DE VALENCIA

\section{Resumen}

Introducción: La artroplastia total de rodilla es una de las cirugías más realizadas en ortopedia y su incidencia está en aumento, siendo la indicación principal en patologías como la artrosis y la artritis reumatoide cuando éstas no responden al tratamiento conservador. Además, en los últimos años está tomando especial relevancia la opinión y satisfacción de los pacientes respecto a la intervención, siendo éstos parte esencial en el proceso asistencial.

Material y métodos: En el presente trabajo se ha realizado un estudio con 30 pacientes intervenidos de artroplastia total de rodilla. Éstos han respondido a dos encuestas, una de ellas acerca de la importancia que otorgaban a distintos aspectos relacionados con el dolor, la movilidad y la vida social antes de la operación, y otra valorando el grado de mejoría que habían experimentado tras la intervención. Posteriormente se ha elaborado un análisis de la frecuencia de respuesta, así como un estudio sobre la existencia de diferencias significativas entre la importancia preoperatoria y la mejoría postoperatoria.

Resultados: Se ha observado que, para los pacientes, los aspectos más importantes antes de la intervención son "no tener ningún dolor", "mejorar su bienestar general" y "mejorar la movilidad". Asimismo, los aspectos que más mejoran tras la intervención son aquellos relacionados con el dolor, así como tener más movilidad en la rodilla. No se han encontrado diferencias estadísticamente significativas entre la importancia de cada expectativa y la mejoría posterior.

Palabras clave: Artroplastia total de rodilla, expectativa del paciente

\section{Summary}

Introduction: Total knee arthroplasty is one of the most performed orthopedic surgeries and its incidence is increasing, being the main indication in pathologies such as osteoarthritis and rheumatoid arthritis when these do not respond to conservative treatment. In addition, in recent years the opinion and satisfaction of patients regarding the intervention has become particularly important, being an essential part of the care process.

Material and methods: A study has been carried out in the present work with 30 patients undergoing surgery for total knee arthroplasty. They responded to two surveys, one on the importance they attached to different aspects related to pain, mobility and social life before the operation, and another on the degree of improvement they had experienced after the intervention. Subsequently, an analysis of the frequency of response has been prepared, as well as a study on the existence of significant differences between preoperative importance and postoperative improvement. Results: It has been observed that, for patients, the most important aspects before the intervention are "to have no pain", "to improve their general well-being" and "to improve mobility". Also, the aspects that improve most after the intervention are those related to pain, as well as having more mobility in the knee. No statistically significant differences have been found between the importance of each expectation and the subsequent improvement.

\author{
Correspondencia: \\ Damián Mifsut Miedes \\ Departamento de Cirugía \\ Universitat de Valencia \\ Avenida Blasco Ibañez 15 \\ 46010, Valencia
}

Email: mifsut.dam@gmail.com 


\section{Introducción}

La expectativa, definida desde un punto de vista médico, es la espera de algo que se cree que se debe de obtener o que tiene que ocurrir. Así, en la práctica, la aplicación del concepto de expectativa no es uniforme y en pocos estudios se define de manera rigurosa. La mayoría utilizan las expectativas de los pacientes como "expectativas de probabilidad" o "expectativas de valores".

La formación de la expectativa del paciente se produce antes de la atención médica y se va modificando durante el transcurso de la misma, pudiendo ser ésta concreta o inespecífica ${ }^{1}$.

En cuanto al contenido de la expectativa del paciente, tiene varias dimensiones, las estructuras sanitarias, entendido como el entorno físico, el personal y la política organizativa de la atención sanitaria, el proceso que engloba a los médicos y personal sanitario tanto en las capacidades técnicas como en la atención interpersonal y por último los resultados que son los efectos o consecuencias finales de la atención.

La importancia de las expectativas radica en intentar entender y satisfacer las necesidades de los pacientes que es uno de los objetivos de la Medicina, y así intentar cumplir dichas expectativas para producir un mayor grado de satisfacción con la atención recibida, conseguir por parte del paciente un mejor seguimiento de las indicaciones médicas, menos cambios de médico y menos demandas ${ }^{2}$.

Desde una perspectiva de política sanitaria resulta importante para medir la calidad de la atención, la distribución de los servicios y el coste de la atención sanitaria.

En la Cirugía Ortopédica las expectativas del paciente pueden afectar la manera en la que éste enfoca y valora su tratamiento, repercutiendo en la indicación quirúrgica. En muchos casos, los pacientes candidatos a cirugía de reemplazo articular tienen expectativas poco realistas de recuperación, en lo que se refiere a dolor y funcionalidad, y ello conduce a altos niveles de insatisfacción con los resultados del procedimiento.

En cuanto a la manera de medir o valorar las expectativas del paciente hay consenso entre los estudios, realizando la medición mediante encuestas que responden los pacientes. Las encuestas que se utilizan pueden ser tipo Likert de 3 a 5 ítems o escalas de puntuación del 1 al 10.

Para la medición de las expectativas de la artroplastia total de rodilla se suele utilizar la Hospital for Special Surgery Knee Replacement Expectations Survey ${ }^{3}$, encuesta realizada en el año 2001 en la que fueron seleccionados 17 ítems que los pacientes que iban a ser intervenidos de artroplastia total de rodilla tenían que puntuar en función de la intensidad de la expectativa.
El objetivo principal de este estudio fue conocer las expectativas de los pacientes ante una artroplastia total de rodilla, centrado en la importancia que tienen éstas antes de la intervención y el grado de mejoría percibido tras la operación.

Los objetivos secundarios fueron:

- $\quad$ Establecer cuáles eran las expectativas más importantes para los pacientes

- Determinar en qué aspectos encontramos una mayor mejoría

Analizar si existían diferencias estadísticas entre la opinión de los pacientes y el grado de mejoría.

\section{Material y Métodos}

Se realizó un estudio transversal en 30 pacientes intervenidos de artroplastia total de rodilla en nuestro Centro, con un tiempo de evolución de más de un año, y que acudieron a consulta de revisión de su operación de artroplastia total de rodilla entre enero y marzo 2017.

Se realizó una adaptación de la encuesta Hospital for Special Surgery Knee Replacement Expectations Survey que consistía en 16 ítems agrupados en tres grupos: dolor, movilidad y vida social.

A los pacientes que acudían a la consulta se les explicaba en qué consistía el trabajo, se les entregaba una carta con información sobre el estudio y dos encuestas tipo Likert acerca de su intervención que debían responder en ese momento:

- $\quad$ En la primera encuesta (Fig. 1) debían valorar la importancia que tenía para ellos cada una de las posibles expectativas, respondiendo a cada uno de los ítems si los consideraban poco importante, bastante importante o muy importante.

\begin{tabular}{|c|c|c|c|c|}
\hline & & $\begin{array}{c}\text { Poco } \\
\text { importante }\end{array}$ & $\begin{array}{c}\text { Bastante } \\
\text { importante }\end{array}$ & $\begin{array}{c}\text { Muy } \\
\text { importante }\end{array}$ \\
\hline \multirow{3}{*}{ 웅 } & No tener ningún dolor & & & \\
\hline & $\begin{array}{l}\text { No tener dolor en reposo aunque } \\
\text { me duelaun poco al andar }\end{array}$ & & & \\
\hline & Mejorar mi bienestar general & & & \\
\hline & & & & \\
\hline \multirow{8}{*}{ 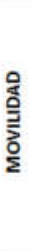 } & Tener más movilidaden la rodilla & & & \\
\hline & Caminar sin bastones & & & \\
\hline & Poder subir y bajar escaleras & & & \\
\hline & Usar transporte público & & & \\
\hline & Poderarrodillarme & & & \\
\hline & Conducir & & & \\
\hline & Más capacidad para el trabajo & & & \\
\hline & $\begin{array}{l}\text { Más capacidad para las actividades } \\
\text { habituales de casa }\end{array}$ & & & \\
\hline & & & & \\
\hline \multirow{5}{*}{ 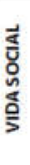 } & Dar largos paseos & & & \\
\hline & Bailar & & & \\
\hline & Hacer gimnasia & & & \\
\hline & Mejorar mi vida sexual & & & \\
\hline & $\begin{array}{l}\text { Hacer trabajos de jardineriao } \\
\text { bricolaje }\end{array}$ & & & \\
\hline
\end{tabular}

Figura 1. Encuesta $n^{0} 1$. 
En la segunda encuesta (Fig. 2) debían de valorar los mismos ítems que en la anterior, pero seleccionando la respuesta en función del grado de mejoría que habían experimentado tras la operación desde nada de mejoría hasta un $100 \%$.

\begin{tabular}{|c|c|c|c|c|c|c|}
\hline & & & & & \\
\hline & & Nada & $25 \%$ & $50 \%$ & $75 \%$ & $100 \%$ \\
\hline \multirow{3}{*}{$\begin{array}{l}\text { 용 } \\
\text { वे }\end{array}$} & $\begin{array}{l}\text { No tener dolor en reposo aunque } \\
\text { me duela un poco al andar }\end{array}$ & & & & & \\
\hline & No tener ningún dolor & & & & & \\
\hline & Mejorar mi bienestar general & & & & & \\
\hline & & & & & & \\
\hline \multirow{8}{*}{ 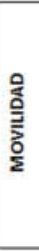 } & Tener más movilidad en la rodilla & & & & & \\
\hline & Caminar $\sin$ bastones & & & & & \\
\hline & Poder subir $y$ bajar escaleras & & & & & \\
\hline & Usar transporte público & & & & & \\
\hline & Poder arrodillarme & & & & & \\
\hline & Conducir & & & & & \\
\hline & Más capacidad para el trabajo & & & & & \\
\hline & $\begin{array}{l}\text { Más capacidad para las actividades } \\
\text { habituales de casa }\end{array}$ & & & & & \\
\hline \multirow{6}{*}{ 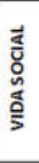 } & & & & & & \\
\hline & Dar largos paseos & & & & & \\
\hline & Bailar & & & & & \\
\hline & Hacer gimnasia & & & & & \\
\hline & Mejorar mi vida sexual & & & & & \\
\hline & $\begin{array}{l}\text { Hacer trabajos de jardineríao } \\
\text { bricolaje }\end{array}$ & & & & & \\
\hline
\end{tabular}

Figura 2. Encuesta $n^{\circ} 2$.

Posteriormente en los resultados se analizaron las frecuencias de respuesta de cada encuesta y se realizó un estudio estadístico con el programa SPSS para encontrar diferencias entre la importancia preoperatoria y el grado de mejoría posterior de cada ítem en particular.

\section{Resultados}

Se recogieron un total de 30 encuestas de pacientes intervenidos mediante ATR en nuestro centro, con un tiempo de evolución mínimo de un año.

En primer lugar se valoraron las expectativas de los pacientes en cuanto a dolor, movilidad y vida social. En la mayoría de casos (16/30) el factor más "importante o muy importante" valorado por los pacientes fue el dolor, en segundo lugar la movilidad y en tercero la vida social (Fig. 3).

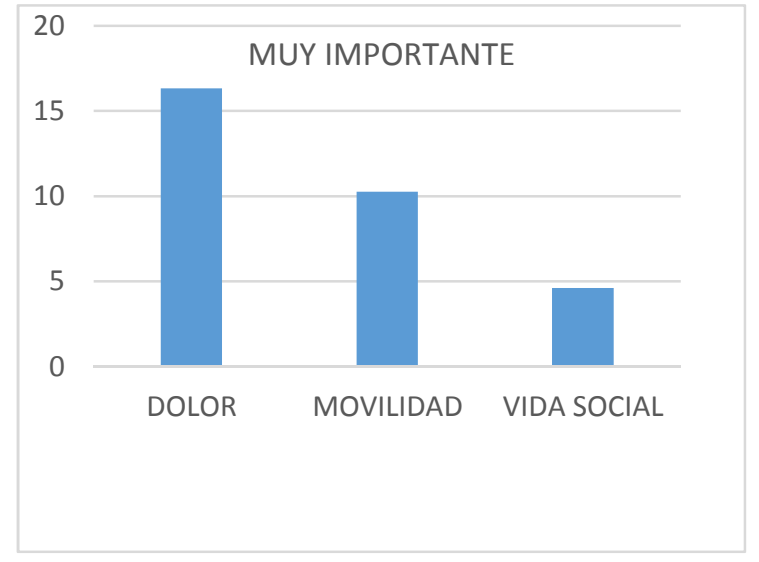

Figura 3. Media de frecuencia de respuesta "muy importante" por grupos.
Resultados de la primera encuesta:

Se valoró cuántos pacientes le dieron la importancia máxima, marcando como muy importante cada uno de los ítems estudiados, de modo que, no tener ningún dolor, mejorar su bienestar general, tener más movilidad en la rodilla y poder subir y bajar escaleras, fueron los valores mejor considerados (Fig. 4).

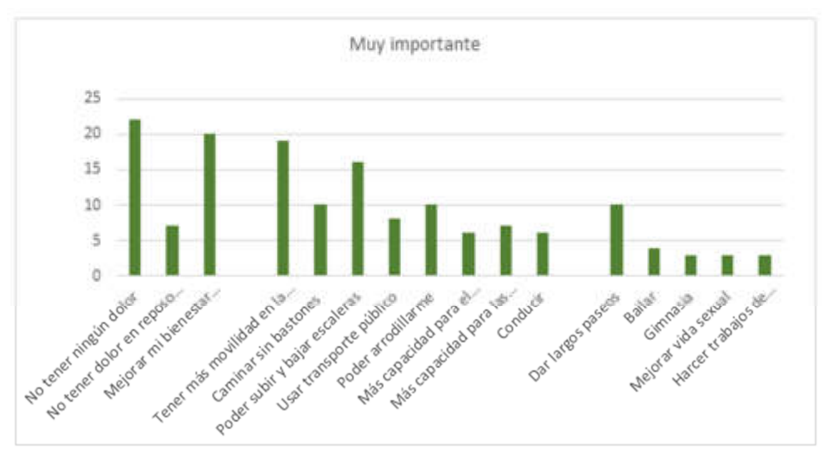

Figura 4. Frecuencia de respuesta muy importante en cada ítem.

En el apartado de dolor, concretamente en "no tener ningún dolor" destaca que para todos los encuestados era un aspecto importante, siendo para el $26,7 \%$ bastante importante y para el 73 ' $3 \%$ muy importante, a ninguno les pareció un aspecto poco importante.

En la variable de "no tener dolor en reposo aunque les duela un poco al andar" también es considerado importante por la mayoría de encuestados, distribuyéndose los resultados en $23{ }^{\prime} 3 \%$ muy importante, $50 \%$ bastante importante y $23,3 \%$ poco importante.

Por último, a la pregunta acerca de la "mejora del bienestar general" vemos que para el $10 \%$ es poco importante, $20 \%$ bastante importante y $66.7 \%$ muy importante. En estos dos últimos ítems un 3'3\% de los pacientes no respondieron.

En el cómputo global, este apartado resulta muy importante para los pacientes siendo ésta su respuesta en un $54{ }^{\prime} 4 \%$ de los casos, bastante importante en un 32 '2\% y sólo un 11 '11\% poco importante (Fig. 5).

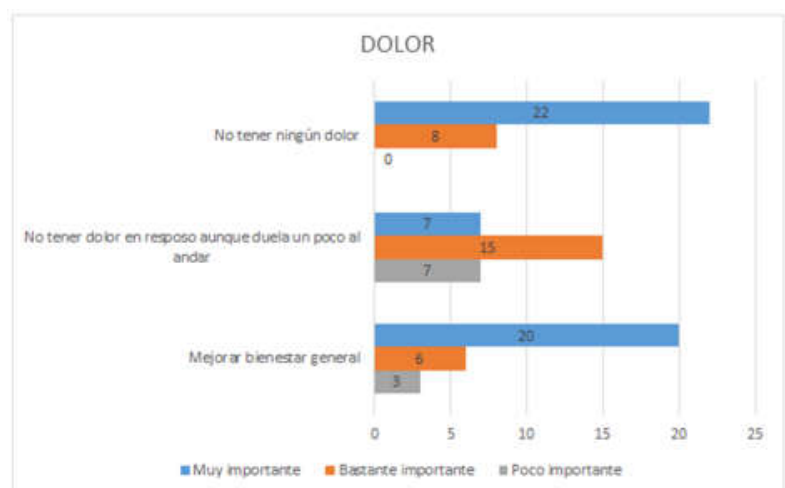

Figura 5. Respuestas de los pacientes en la encuesta sobre importancia preoperatoria al apartado del dolor. 
En cuanto a la movilidad, "tener más movilidad en la rodilla" para el 96 ' $7 \%$ de los encuestados les parecía importante, siendo muy importante para el 63'3\% y bastante importante para el 33'3\%. Sólo uno de los encuestados que representa el 3'3\% valoró este aspecto como poco importante, el resto de valores se muestran en la Figura 6.

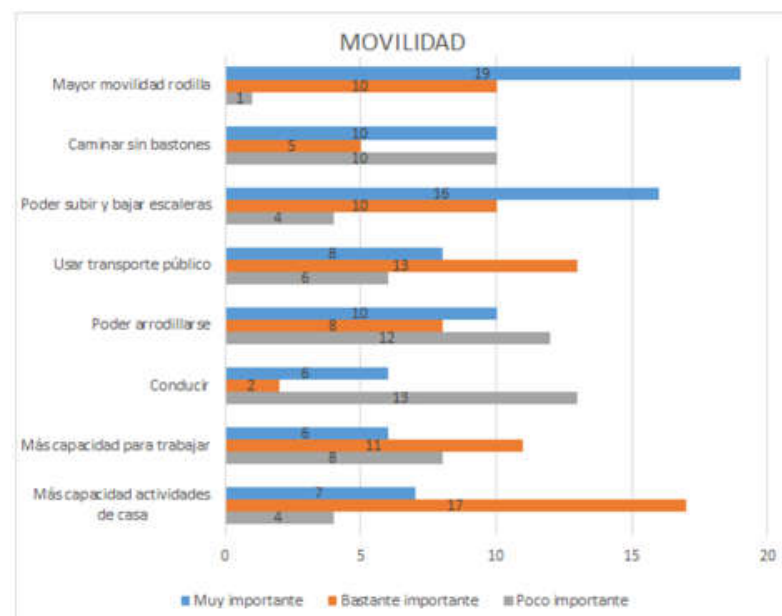

Figura 6. Respuestas de los pacientes en la encuesta sobre importancia preoperatoria al apartado de movilidad.

En último lugar los pacientes debían valorar actividades relacionadas con su vida social

y que realizan habitualmente. Dentro de éste grupo lo más importante para los pacientes fue el poder "realizar largos paseos", el 33'3\% respondió que para ellos era muy importante y para el $50 \%$ bastante importante. El $167 \%$ restante lo consideró poco importante.

El resto de actividades se muestran en la Figura 7.

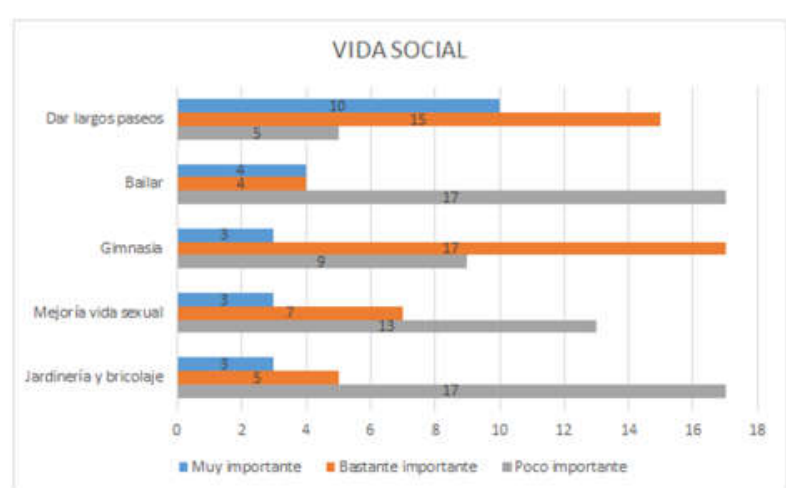

Figura 7. Respuestas de los pacientes en la encuesta sobre importancia preoperatoria al apartado de vida social.

\section{Resultados de la segunda encuesta:}

Posteriormente se procedió al análisis de frecuencias de los resultados de la segunda encuesta, en la que los pacientes respondían acerca de cuanto valoraban lo que habían mejorado tras la operación en cada uno de los ítems.
En el apartado de dolor, la media de mejoría de los tres grupos supera el $85 \%$, dando a entender la gran satisfacción de los pacientes en este aspecto (Tabla I).

\begin{tabular}{|l|r|r|r|}
\cline { 2 - 4 } \multicolumn{1}{c|}{} & \multicolumn{1}{c}{$\begin{array}{c}\text { No tener ningún } \\
\text { dolor }\end{array}$} & $\begin{array}{c}\text { No tener dolor en } \\
\text { reposo aunque duela } \\
\text { un poco al andar }\end{array}$ & $\begin{array}{c}\text { Mejorar bienestar } \\
\text { general }\end{array}$ \\
\hline Válidos & 30 & 29 & 29 \\
Perdidos & 0 & 1 & 1 \\
Media & $85,83 \%$ & $86,21 \%$ & $89,66 \%$ \\
\hline
\end{tabular}

Tabla I. Media de mejoría experimentada por el paciente tras la intervención en respuesta al apartado de dolor.

Los encuestados cuando fueron preguntados si tras la operación "no tenían ningún dolor", la respuesta fue que mejoraron todos al menos un $50 \%$. Concretamente un 13 '3\% respondió $50 \%$, un $30 \%$ mejoría de un $75 \%$ y la mayor proporción manifestó haber experimentado una mejora completa (56'7\%). Se muestran en la Figura 6 el resto de valores analizados.

Siguiendo con el análisis de las encuestas, en la valoración de la mejora de la movilidad de los pacientes. Encontramos aquí gran variabilidad entre la media de mejoría de cada aspecto, como se aprecia en la Tabla II "tener más movilidad en la rodilla" es el que mayor recuperación presenta, y en el lado contrario, "poder arrodillarse", es el ítem en el que menos mejoran.

\begin{tabular}{|c|r|r|r|r|r|r|r|r|}
\multicolumn{10}{c|}{ Estadisticos } \\
\cline { 2 - 9 } & $\begin{array}{c}\text { Tener } \\
\text { más } \\
\text { movilidad } \\
\text { en la } \\
\text { rodilla }\end{array}$ & $\begin{array}{c}\text { Caminar } \\
\text { sin } \\
\text { bastón }\end{array}$ & $\begin{array}{c}\text { Subir y } \\
\text { bajar } \\
\text { escaleras }\end{array}$ & $\begin{array}{c}\text { Usar } \\
\text { transponte } \\
\text { público }\end{array}$ & $\begin{array}{l}\text { Poderse } \\
\text { arrodillar }\end{array}$ & Conducir & $\begin{array}{c}\text { Capacidad } \\
\text { para el } \\
\text { trabajo }\end{array}$ & $\begin{array}{c}\text { Capacidad } \\
\text { para las } \\
\text { actividad } \\
\text { de casa }\end{array}$ \\
\hline Válidos & 30 & 24 & 30 & 25 & 19 & 19 & 26 & 29 \\
Perdidos & 0 & 6 & 0 & 5 & 11 & 11 & 4 & 1 \\
Media & $85,83 \%$ & $78,13 \%$ & $72,50 \%$ & $82,00 \%$ & $23,28 \%$ & $65,79 \%$ & $72,12 \%$ & $79,31 \%$ \\
\hline
\end{tabular}

Tabla II. Media de mejoría experimentada por el paciente tras la intervención en respuesta al apartado de movilidad.

La "movilidad en la rodilla" ha tenido una media de mejora de $85{ }^{\prime} 83 \%$, siendo el valor mínimo de mejora del $50 \%$. La mitad de los pacientes creen haber sentido una recuperación completa, el 43 '3\% del $75 \%$ y un 6 ' $7 \%$ del $50 \%$.

En el análisis de frecuencia con respecto al grado de mejoría experimentado en actividades relacionadas con el estilo de vida, se observa en general que las medias de mejora son más bajas. En la Tabla III podemos observar que "dar largos paseos" tiene una media elevada de mejora muy cerca del $80 \%$, y en "gimnasia" tiene una mejora del 62 '96\%. Los otros tres ítems valorados no experimentan ni un $50 \%$ de mejora media, éstos son "bailar", "mejorar la vida sexual" y "realizar trabajos de jardinería y bricolaje" que tienen de media $45^{\prime} 65 \%, \quad 33^{\prime} 33 \%$ y $45^{\prime} 65 \%$ respectivamente. 


\begin{tabular}{|l|r|r|r|r|r|}
\hline & $\begin{array}{c}\text { Dar largos } \\
\text { paseos }\end{array}$ & Bailar & Gimnasia & $\begin{array}{c}\text { Mejorar vida } \\
\text { sexual }\end{array}$ & $\begin{array}{c}\text { Hacer trabajos } \\
\text { de jardineria o } \\
\text { bricolaje }\end{array}$ \\
\hline Válidos & 30 & 23 & 27 & 21 & 23 \\
Perdidos & 0 & 7 & 3 & 9 & 7 \\
Media & $79,17 \%$ & $45,65 \%$ & $62,96 \%$ & $33,33 \%$ & $45,65 \%$ \\
\hline
\end{tabular}

Tabla III. Media de mejoría experimentada por el paciente tras la intervención en respuesta al apartado de vida social.

En la Figura 8 se pueden observar las medias de mejora de cada grupo general, en la que destaca que en el aspecto que más mejoran es en el dolor con una media de $87^{\prime} 23 \%$, seguido de la movilidad con $69^{\prime} 87 \%$ y en último lugar de la vida social que tiene de promedio 53 '35\%.

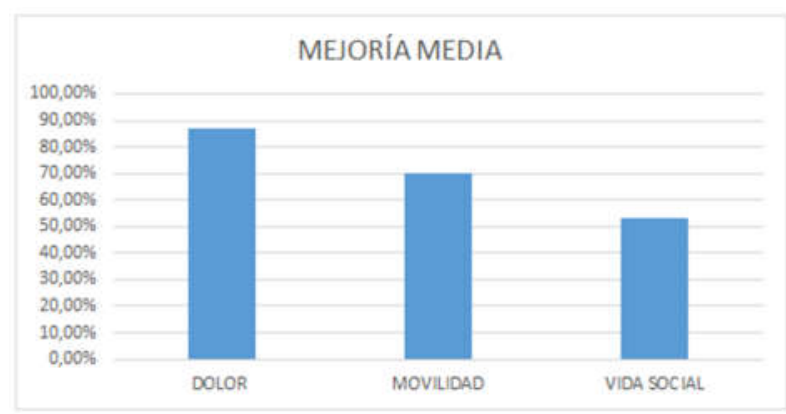

Figura 8: Grado de mejoría media experimentada por los pacientes tras la intervención por apartados.

Asimismo, se realizó un análisis estadístico, para valorar si existen diferencias significativas entre la importancia que tenía cada variable para los pacientes y el grado de mejoría experimentado después. Para ello, se recodificaron las variables en variables dicotómicas y se utilizó el estadístico exacto de Fisher. La variable sobre la importancia que tenía cada aspecto se agrupó en dos opciones, muy importante y no muy importante, ésta última agrupaba las opciones de bastante y poco importante. La variable sobre la mejoría tenía dos valores, poca mejoría compuesto por las mejorías anteriores de 0,25 y $50 \%$, y mucha mejoría formado por 75 y $100 \%$.

Las hipótesis que pretendíamos resolver con esta prueba eran:

HO: No existen diferencias estadísticamente significativas entre la importancia y el grado de mejoría

- $\quad \mathrm{H} 1$ : Existen diferencias estadísticamente significativas entre la importancia y el grado de mejoría

Con estas pruebas se pudo afirmar que en el presente estudio, la importancia que le dieron los pacientes a cada aspecto antes de la operación, no influyó en el grado de mejoría experimentado posteriormente.

\section{Discusión}

La importancia de la artroplastia de rodilla ha ido en aumento y se prevé que su incidencia continúe éste progreso, su eficacia está demostrada y los criterios médicos para realizar la intervención están bien establecidos. Pero es importante conocer la opinión de los pacientes, dado que van a ser ellos quienes se van a someter al proceso. Por ello, hay que tener en cuenta sus expectativas, cuanta importancia tienen para ellos algunos aspectos de la vida que esperan mejorar y en cuales experimentan una mayor mejoría. Estos resultados pueden ser muy útiles y podrían ser usados como parte de la información preoperatoria al paciente para aumentar el nivel de conciencia y cooperación, así como facilitar la rehabilitación.

Hay que tener en cuenta, como señala Eisler et al. ${ }^{4}$ que las expectativas de los pacientes pueden ser elevadas, porque el hecho de tener expectativas anima a la operación, es decir, la gente que decide intervenirse tiene unas expectativas altas, en cambio la gente que declina esta opción tiene expectativas bajas y se decanta por tratamientos más conservadores.

Otro motivo por el que resulta interesante conocer las opiniones de los pacientes radica en que la opinión entre el cirujano y el paciente puede no ser la misma. Como vemos en un estudio realizado en España ${ }^{5}$, las opiniones coinciden sólo en el $62 \%$ de los casos, pero en el $38 \%$ restante no, siendo el dolor y la deambulación peor valoradas por el paciente que por el cirujano que lo había intervenido.

No se han realizado comparaciones entre grupos de edad, debido a que se ha observado que la edad no es un factor que determine peores resultados funcionales ${ }^{6}$.

Tampoco se han estudiado las diferencias entre sexos $u$ otras variables demográficas, porque en un estudio reciente, se concluyó que la expectativa preoperatoria no está influenciada por la demografía del paciente o la función preoperatoria ${ }^{7}$.

En dicho estudio también observaron que las expectativas más altas predicen mayor mejora postoperatoria, por tanto, queda clara la relación de las expectativas en el resultado postoperatorio. A pesar de esto en nuestro estudio no hemos encontrado diferencias estadísticamente significativas entre los pacientes que no les parecía muy importante las expectativas y los que sí que las consideraban muy importantes, entendiendo que a pesar de que tener expectativas da un mejor resultado final, éste no está relacionado con la importancia que les dan a las expectativas los pacientes. En nuestro estudio, como se ha visto con anterioridad, los aspectos generales a que mayor valor otorgan los pacientes son en primer lugar al alivio del dolor, en segundo la movilidad y en tercero algunos aspectos de la vida social.

Los ítems considerados más importantes para los pacientes antes de someterse a la operación eran "no tener ningún dolor", "mejorar el bienestar general", "tener mayor movilidad en la rodilla" y "poder subir y bajar escaleras". Estos resultados son similares a los observados en otras publicaciones como la de Eisler et al. $^{4}$ que, aunque fue realizado en pacientes sometidos a 
artroplastia de cadera, observa que el 92\% espera mejorar el dolor y el $82 \%$ mejorar su capacidad para caminar. Siguiendo en esta misma línea encontramos el estudio de Mahomed et al. ${ }^{8}$, realizado a pacientes intervenidos de artroplastia de cadera o rodilla y cuestionados sobre sus expectativas prequirúrgicas, en el que el $75 \%$ de los pacientes espera no tener ningún dolor y el $45 \%$ no tener ningún tipo de limitación a la actividad habitual. También vemos el estudio de Lingard et al. ${ }^{9}$, que evaluaba las diferencias entre las expectativas de distintos países ante una artroplastia total de rodilla, y en el que el $70 \%$ de los encuestados espera una mejora total del dolor y un $25 \%$ una mejora significativa, aunque no sea total. En otro trabajo similar $^{10}$ el $95 \%$ espera alguna mejora de dolor y el $88 \%$ que ésta sea completa, todos los pacientes desean mejorar su bienestar funcional y el $90 \%$ una mejora de las funciones básicas.

A la hora de valorar cuanto habían mejorado los pacientes, basándonos en las medias de cada apartado, en el que más mejoran los pacientes es en el de dolor, experimentando una mejoría del $87 \%$. Siendo el dolor uno de los motivos por el que los pacientes son intervenidos y la importancia que le otorgan los pacientes, es significativo observar que el grado de mejoría es muy elevado. Podemos ver que este resultado es similar al de otros estudios como el realizado por Pagès et al. ${ }^{11}$ que valoró la satisfacción de los pacientes tras 5 años de la intervención y el 88 '5\% afirmaba que el dolor era inexistente o ligero. En el mismo artículo, el grado de satisfacción con la marcha alcanzó el $73{ }^{\prime} 37 \%$, muy parecido también al obtenido en nuestro estudio en el apartado de movilidad, en el que la media de los 8 ítems era de $69^{\prime} 87 \%$, existiendo en este grupo una gran variabilidad entre cada aspecto.

En cuanto al tema de la vida social, la mejoría media de los pacientes es de 53'35\%. Aquí se deberían buscar las causas de la poca mejoría, pudiendo estar relacionado con la poca importancia que dan los pacientes a los ítems de este apartado como "bailar", "mejorar la vida sexual" o "hacer trabajos de jardinería y bricolaje" o que sean actividades que no les gusten o no realicen.
Hay que tener en mente que los pacientes son mayores y suelen tener otras patologías asociadas que pueden influir en la mejoría observada tras la intervención.

En los que menos mejoría se observa son: "poder arrodillarse", "mejorar vida sexual", "bailar" y "hacer trabajos de jardinería y bricolaje". Teniendo esto en consideración, habría que informar a los pacientes antes de la intervención de la dificultad que encontrarán para realizar dichas acciones.

De éstos sólo "poder arrodillarse" no era uno de los considerados menos importantes y por tanto llama la atención la poca mejoría que encuentran los pacientes para realizar esta acción, siendo esta mejoría menor del $25 \%$. Además, cabría preguntarse el motivo por el cuál no pueden arrodillarse, si se debe a una insuficiencia funcional o si en cambio guarda relación con la cicatriz quirúrgica, si la causa fuera ésta última podrían plantearse otros abordajes quirúrgicos para obtener un mayor grado de mejoría. Las otras estaban valoradas también como menos importantes, y aunque para muchos son acciones que no realizan o con poco peso en su vida, para los que sí que las consideran importantes podría ser un problema la escasa mejoría tras la intervención.

\section{Conclusiones}

- Las expectativas más importantes para los pacientes antes de ser intervenidos de artroplastia total de rodilla son no tener ningún dolor, mejorar su bienestar general y tener mayor movilidad en la rodilla.

- $\quad$ El dolor es el apartado que mayor grado de mejoría presenta tras la artroplastia total de rodilla.

- No existen diferencias estadísticamente significativas entre la importancia que tiene para los pacientes la expectativa antes de operarse y el grado de mejoría que experimentan después. 
FERRANDO-MESEGUER EJ Y COLS: Expectativas del paciente ante la artroplastia total de rodilla.

\section{Bibliografía}

1. Redman, R. and Lynn, M. (2005). Assessment of Patient Expectations for Care. Research and Theory for Nursing Practice, 19(3), 275-285.

2. Kravitz, R. (1996). Patients' Expectations for Medical Care: An Expanded Formulation Based on Review of the Literature. Medical Care Research and Review, 53(1), 3-27.

3. Mancuso, C., Sculco, T., Wickiewicz, T., Jones, E., Robins, L., Warren, R., \& Williams-Russo, P. (2001). Patients' Expectations of Knee Surgery. The Journal Of Bone And Joint Surgery, 83, 1005-1012.

4. Eisler, T., Svensson, O., Tengström, A., \& Elmstedt, E. (2002). Patient expectation and satisfaction in revision total hip arthroplasty. The Journal Of Arthroplasty, 17(4), 457-462.

5. Hernández-Vaquero, D., Cervero-Suárez, J., de Cima-Suárez, M., Cuervo-Olay, M., \& Fernández-Carreira, J. (2008). Resultados de las artroplastias de rodilla ¿Coinciden la opinión del cirujano y la del paciente? Revista Española De Cirugía Ortopédica Y Traumatología, 52(5), 295-299.

6. Moreno Palacios, J., Cátedra Vallés, E., Plazas Andreu, N., Sancho Loras, R., Manjón-Cabezas Subirats, J., \& Mozo Muriel, Á. (2009). Comparación de resultados en función de la edad en pacientes ancianos con artroplastia total de rodilla. Revista Española De Geriatría Y Gerontología, 44(3), 120-123.

7. Jain, D., Nguyen, L., Bendich, I., Nguyen, L., Lewis, C., \& Huddleston, J. et al. (2017). Higher Patient Expectations Predict Higher Patient-Reported Outcomes, But Not Satisfaction, in Total Knee Arthroplasty Patients: A Prospective Multicenter Study. The Journal Of Arthroplasty

8. Mahomed, N., Liang, M., Cook, E., Daltroy, L., Fortin, P., Fossel, A., \& Katz, J. (2002). The Importance of Patient Expectations in Predicting Functional Outcomes After Total Joint Arthroplasty. The Journal Of Rheumatology, 29(6), 1273-1279.

9. Lingard, E., Sledge, C., Learmonth, I., \& Kinemax Outcomes Group,. (2006). Patient Expectations Regarding Total Knee Arthroplasty: Differences Among the United States, United Kingdom, and Australia. The Journal Of Bone And Joint Surgery, 88(6), 1201-1207.

10. Muniesa Portolés, J. (2006). Anàlisi de les expectatives dels pacients candidats a artroplàstia total de genoll. Doctorado. Universitat Autònoma de Barcelona, Facultat de Medicina, Departament de Cirurgia.

11. Pagès, E., Iborra, J. and Cuxart, A. (2004). Prótesis total de rodilla estándar. Análisis de supervivencia del implante a los 5 años. Rehabilitación, 38(1), 7-12. 